\title{
Relationship between the cholesterol ester transfer protein TaqIB polymorphism and the lipid-lowering effect of atorvastatin in patients with coronary atherosclerotic heart disease
}

\author{
J. Li ${ }^{1}$, L. Zhang', N.Z. Xie', B. Deng ${ }^{2}$, L.X. Lv ${ }^{3}$ and L.Q. Zheng ${ }^{3}$ \\ ${ }^{1}$ Department of Cardiology, Tongji Hospital, Tongji University, \\ Shanghai, China \\ ${ }^{2}$ Department of Cardiology, Longhua Hospital, \\ Shanghai University of Traditional Chinese Medicine, Shanghai, China \\ ${ }^{3}$ Department of Biochemistry, Medical School of Tongji University, \\ Shanghai, China
}

Corresponding author: J. Li

E-mail: jianlicnen@163.com

Genet. Mol. Res. 13 (1): 2140-2148 (2014)

Received January 28, 2013

Accepted September 29, 2013

Published March 24, 2014

DOI http://dx.doi.org/10.4238/2014.March.24.21

ABSTRACT. This study aimed to investigate the relationship between
the cholesterol ester transfer protein (CETP) gene TaqIB polymorphism
and the lipid-lowering effect of atorvastatin in patients with coronary
atherosclerotic heart disease. Two hundred eighty-eight patients were
divided into a control group, an acute coronary syndrome (ACS) group,
and a stable coronary heart disease (CHD) group. Blood biochemical
indices were determined using the enzyme method, and polymerase
chain reaction-restriction fragment length polymorphism (PCR-RFLP)
analysis was performed to study the TaqIB polymorphism of the CETP
gene. The ACS and stable CHD groups were treated with atorvastatin,
and blood lipid levels were reexamined after three months. Plasma
levels of total cholesterol (TC), low-density lipoprotein cholesterol
(LDL-C), and lipoprotein(a) were all significantly higher in the ACS 
and stable CHD groups compared to the control group $(\mathrm{P}<0.05$ or $\mathrm{P}<0.01)$. After three months of treatment with atorvastatin, plasma levels of TC, LDL-C, triglycerides (TG) (only in patients with genotype B2B2), and lipoprotein(a) (only in patients with genotype B1B2) were all significantly decreased $(\mathrm{P}<0.05$ or $\mathrm{P}<0.01)$. After treatment, the plasma level of TG was lower in patients with genotype B2B2 compared to patients with genotypes $\mathrm{B} 1 \mathrm{~B} 1$ or $\mathrm{B} 1 \mathrm{~B} 2$ (B1 carriers) $(\mathrm{P}<$ 0.01). Therefore, the CETP TaqIB polymorphism is associated with the lipid-lowering effect of atorvastatin in patients with CHD.

Key words: Cholesterol ester transfer protein; Gene; Atorvastatin; Coronary artery disease

\section{INTRODUCTION}

Coronary atherosclerotic heart disease has become one of the major diseases threatening human health. Acute coronary syndrome (ACS) is caused by incomplete or complete coronary obstruction due to the rupture of unstable atherosclerotic plaque in the coronary artery, and its clinical manifestations include unstable angina, acute myocardial infarction, and sudden death (Pillois et al., 2009; Ranjith et al., 2009). Intervention of risk factors for coronary heart disease (CHD) has increasingly attracted attention from medical workers. Dyslipidemia is a recognized important risk factor for CHD. Previous studies (Hsieh et al., 2007; Tsujita et al., 2007; Kimura, 2009; Estévez-González et al., 2010) have demonstrated that the cholesterol ester transfer protein (CETP) plays a vital role in lipid metabolism. Expression of the CETP gene determines its function, thereby affecting lipid metabolism and CHD occurrence and development. Statins can reduce the low-density lipoprotein cholesterol (LDL-C) level in blood, and thus increase the incidence and mortality rate of cardiovascular and cerebrovascular events. Therefore, whether subtle relationships exist among CETP, statins, and blood lipids deserves further investigation. Effects of CETP gene expression on the lipid-lowering effects of statins have not yet been reported. In this study, the blood lipid profiles of 99 patients with stable CHD, 147 patients with ACS, and 42 controls were observed. The relationship between the CETP TaqIB polymorphism and serum lipid levels was analyzed in patients with CHD, and the effect of the CETP TaqIB polymorphism on lipid-lowering effects of atorvastatin was investigated.

\section{MATERIAL AND METHODS}

\section{Subjects}

Two hundred eighty-eight patients who were admitted to our hospital between January 2011 and October 2012 were divided into a control group, an ACS group, and a stable CHD group. This study was conducted in accordance with the Declaration of Helsinki and with approval from the Ethics Committee of Tongji Hospital. Written informed consent was obtained from all participants. In the control group (42 cases, including 28 males and 14 females with an average age of $55.83 \pm 13.05$ years), all patients were admitted with chest pain as their chief complaint, but yielded negative coronary angiography results. These patients 
had not taken lipid-lowering drugs, and hepatic and renal functions were normal. Patients with blood diseases were excluded.

The ACS group ( 147 cases, including 105 males and 42 females with an average age of $56.69 \pm 12.51$ years) comprised 88 cases of unstable angina and 59 cases of acute myocardial infarction. The inclusion criteria were as follows: the time of persistent chest pain was more than 20 minutes; dynamic ST-T changes appeared in the electrocardiogram (ECG); the levels of markers for myocardial injury (creatine kinase and its isoenzyme MB, myoglobin, and troponin) were increased by more than three times (more than two or three of the above events occurred simultaneously). Furthermore, coronary angiography results revealed $\geq 70 \%$ diameter stenosis in at least one of the left anterior descending coronary artery, left circumflex artery, and right coronary artery, with visible unstable plaque or thrombus projection. The blood flow was lower than grade 2 in thrombolysis in myocardial infarction (TIMI) with or without culprit vessels. No patients had taken lipid-lowering drugs or had a history of long-term hormone use.

In the stable CHD group ( 99 cases, including 69 males and 30 females, with an average age of $73.66 \pm 15.45$ years), the inclusion criteria were as follows. Patients had a history of exertional angina. Newly diagnosed CHD patients were suspected to have CHD based on ECG, treadmill, blood biochemistry, and echocardiography examination, with a positive coronary arteriography result and negative markers for myocardial injury. No patients had taken lipid-lowering drugs.

The height, weight, and waistline of all patients were measured, and the body mass index (BMI) [weight $(\mathrm{kg}) /$ height $\left.^{2}\left(\mathrm{~m}^{2}\right)\right]$ was calculated.

\section{Blood biochemical indices}

In the ACS group, patients lay in repose, and elbow venous blood was taken within $24 \mathrm{~h}$ after disease onset. In the stable CHD and control groups, elbow venous blood was taken in early morning with supine position. Three milliliters venous blood was used for separation of serum. Total cholesterol (TC), triglyceride (TG), low-density lipoprotein cholesterol (LDL-C), high-density lipoprotein cholesterol (HDL-C), and lipoprotein(a) were detected using the ADVIA1650 automatic biochemical analyzer (kits were provided by COLAB Company, Germany). Fasting blood glucose (FPG) was detected using the hexokinase method. For the ACS and stable CHD groups, 2 $\mathrm{mL}$ fasting venous blood was anticoagulated using edetate disodium (EDTA). After shaking, standing, and demixing, samples were stored at $-20^{\circ} \mathrm{C}$ until used for analysis of CETP TaqIB genotypes.

\section{Analysis of CETP TaqIB genotypes}

After thawing fasting venous blood, the hemolysis method using low-permeability buffer was applied for extraction of genomic DNA from white blood cells. CETP TaqIB gene fragments were amplified using polymerase chain reaction (PCR) with primer 1 (5'-CACTAGCCCAGAGAGAGGAGTGC-3') and primer 2 (5'-CTGAGCCCAGCCGCACA CTAA-3'), respectively. PCR conditions were as follows: $300 \mathrm{mM}$ Tris- $\mathrm{HCl}, 75 \mathrm{mM}$ ammonium sulfate, $12.5 \mathrm{mM} \mathrm{MgCl} 2, \mathrm{pH} 10.0$, degeneration at $95^{\circ} \mathrm{C}$ for $30 \mathrm{~s}$, annealing at $62^{\circ} \mathrm{C}$ for $30 \mathrm{~s}$, and extension at $72^{\circ} \mathrm{C}$ for $60 \mathrm{~s}$ for 35 cycles. The amplified product was digested with TaqI enzyme, and then 2\% agarose gel electrophoresis was conducted for identification of CETP TaqIB genotypes (Figure 1). 


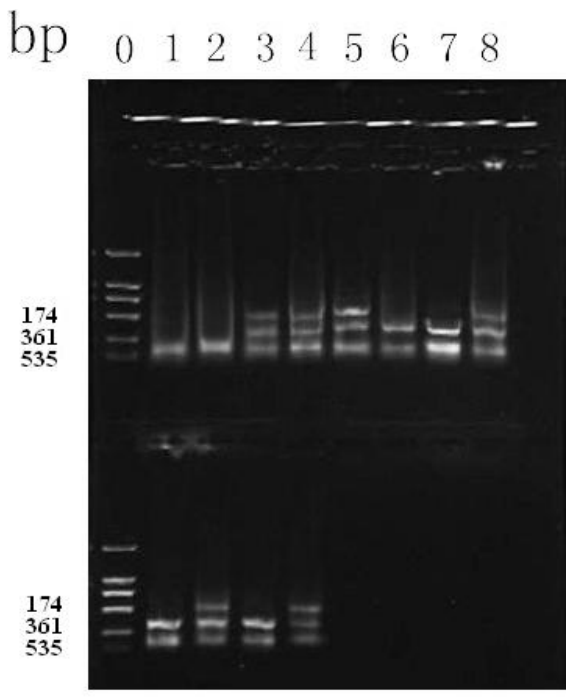

09101112

Figure 1. Enzyme electrophoresis of CETP TaqIB. Lane $0=$ marker; lanes 1 and $2=$ one band (535 bp), defective heterozygote B2B2; lanes $6,7,9$, and $11=$ two bands (361 and $174 \mathrm{bp}$ ), mutant homozygote B1B1; lanes 3, 4, 5, 8,10 , and $12=$ three bands $(535,361$, and $174 \mathrm{bp})$, mutant heterozygote B1B2.

\section{Treatment and follow-up}

After admission, all patients in the ACS and stable CHD groups were treated with statin drugs to lower blood lipid levels. The specific scheme was as follows: $20 \mathrm{mg}$ atorvastatin (Pfizer Inc., USA) was orally administered one time every night. After three months of treatment, outpatient follow-up was performed by telephone appointment, and blood lipid was reexamined using the methods described above.

\section{Statistical analysis}

Data are reported as means \pm SD. Statistical analysis was performed using the SPSS 17.0 statistical software. The Student's $t$-test was used to analyze differences before and after lipid lowering therapy with atorvastatin, and single factor analysis of variance (ANOVA) was conducted for comparing blood lipid profiles among multiple groups. $\mathrm{P}<0.05$ was considered to be statistically significant.

\section{RESULTS}

\section{Related indices comparison}

As shown in Table 1, before lipid-lowering therapy with atorvastatin, the plasma levels of TC, LDL-C, and lipoprotein(a) were significantly higher in both the ACS and stable CHD groups compared to the control group $(\mathrm{P}<0.05$ or $\mathrm{P}<0.01)$. There was no significant 
difference in either TG or HDL-C levels among the three groups. Waistlines were significantly larger in the ACS group compared to the control group $(\mathrm{P}<0.05)$, FPG levels were markedly higher in the ACS and stable CHD groups compared to the control group $(\mathrm{P}<0.05)$, and there was no significant difference in BMI among the three groups.

\begin{tabular}{|c|c|c|c|}
\hline Group & Control group & ACS group & Stable CHD group \\
\hline $\mathrm{N}$ & 42 & 147 & 99 \\
\hline $\mathrm{TC}(\mathrm{mM})$ & $4.18 \pm 0.75$ & $4.67 \pm 1.08 *$ & $4.64 \pm 0.87^{*}$ \\
\hline $\mathrm{TG}(\mathrm{mM})$ & $1.48 \pm 1.03$ & $1.59 \pm 0.98$ & $1.69 \pm 1.56$ \\
\hline HDL-C (mM) & $1.07 \pm 0.33$ & $1.04 \pm 0.29$ & $1.05 \pm 0.26$ \\
\hline LDL-C (mM) & $2.60 \pm 0.64$ & $3.12 \pm 0.93^{* *}$ & $3.00 \pm 0.77 * *$ \\
\hline Lipoprotein(a) (mg/L) & $259.92 \pm 105.15$ & $347.74 \pm 172.37 * *$ & $348.89 \pm 201.08 *$ \\
\hline BMI $\left(\mathrm{kg} / \mathrm{m}^{2}\right)$ & $23.92 \pm 3.23$ & $24.09 \pm 2.95$ & $24.02 \pm 3.01$ \\
\hline Waistline $(\mathrm{cm})$ & $86.10 \pm 9.99$ & $88.52 \pm 8.66^{*}$ & $87.97 \pm 9.02$ \\
\hline FPG $(\mathrm{mM})$ & $5.52 \pm 1.10$ & $6.86 \pm 2.48^{*}$ & $6.22 \pm 2.01^{*}$ \\
\hline
\end{tabular}

$* \mathrm{P}<0.05$ and $* * \mathrm{P}<0.01$ compared with control group.

\section{CETP TaqIB genotype effects}

As shown in Table 2, HDL-C levels in patients with genotype B1B2 were significantly higher compared to those in patients with genotype B1B1 $(\mathrm{P}=0.005)$. There was no statistical difference in any blood lipid index between B1B2 and B2B2 patients.

\section{Table 2. Effects of CETP TaqIB genotypes on blood lipid levels.}

\begin{tabular}{lccr}
\hline Lipid profile index & \multicolumn{3}{c}{ CETP TaqIB genotype } \\
\cline { 2 - 4 } & B1B1 & B1B2 & B2B2 \\
\hline $\mathrm{N}$ & 82 & 73 & 21 \\
TC $(\mathrm{mM})$ & $4.68 \pm 0.93$ & $4.52 \pm 0.89$ & $4.64 \pm 0.99$ \\
TG $(\mathrm{mM})$ & $1.66 \pm 1.02$ & $1.44 \pm 1.05$ & $1.68 \pm 1.27$ \\
HDL-C $(\mathrm{mM})$ & $0.99 \pm 0.23$ & $1.10 \pm 0.32 * *$ & $1.10 \pm 0.27$ \\
LDL-C $(\mathrm{mM})$ & $3.12 \pm 0.79$ & $2.93 \pm 0.75$ & $3.07 \pm 0.79$ \\
Lipoprotein(a) $(\mathrm{mg} / \mathrm{L})$ & $358.35 \pm 191.30$ & $344.64 \pm 175.68$ & $355.38 \pm 213.32$ \\
\hline
\end{tabular}

$* * \mathrm{P}<0.01$ compared with B1B1; DNA specimens of 176 patients in ACS group and stable CHD group were obtained for determination of genotype.

\section{Atorvastatin effects}

After three months of lipid-lowering therapy with atorvastatin, the plasma levels of TC, LDL-C, TG (only in B2B2 patients), and lipoprotein(a) (only in B1B2 patients) all decreased significantly $(\mathrm{P}<0.05$ or $\mathrm{P}<0.01)$ (Table 3$)$.

\section{Association between allele and lipid-lowering effects of atorvastatin}

After 3 months of treatment with atorvastatin, only the plasma level of TG in B2B2 patients significantly decreased $(\mathrm{P}<0.05)$. Furthermore, the plasma level of TG in B2B2 patients was lower than that in $\mathrm{B} 1 \mathrm{~B} 1$ and $\mathrm{B} 1 \mathrm{~B} 2$ patients (B1 carriers) after treatment $(\mathrm{P}=0.009)$. 


\begin{tabular}{|c|c|c|c|c|}
\hline Blood lipid profile & B1B1 & B1B2 & B1 carriers & B2B2 \\
\hline$\overline{\mathrm{N}}$ & 56 & 42 & 98 & 10 \\
\hline TC (before treatment) (mM) & $4.83 \pm 0.96$ & $4.66 \pm 0.90$ & $4.73 \pm 0.92$ & $4.77 \pm 1.12$ \\
\hline TC (after treatment) (mM) & $4.25 \pm 1.03 * *$ & $3.73 \pm 1.00 * *$ & $3.96 \pm 1.01^{* *}$ & $3.70 \pm 0.83^{* *}$ \\
\hline$\Delta \mathrm{TC}(\mathrm{mM})$ & $-0.57 \pm 1.00$ & $-0.92 \pm 1.06$ & $-0.72 \pm 1.03$ & $-1.07 \pm 0.71$ \\
\hline $\mathrm{N}$ & 56 & 42 & 98 & 10 \\
\hline TG (before treatment) (mM) & $1.73 \pm 1.09$ & $1.58 \pm 1.16$ & $1.61 \pm 1.10$ & $2.12 \pm 1.95$ \\
\hline TG (after treatment) (mM) & $1.82 \pm 1.40$ & $1.60 \pm 0.93$ & $1.77 \pm 1.01$ & $1.21 \pm 0.82 *$ \\
\hline$\Delta \mathrm{TG}(\mathrm{mM})$ & $0.10 \pm 1.11^{\Delta \Delta}$ & $0.03 \pm 1.11^{\Delta}$ & $0.07 \pm 1.10^{\Delta \Delta}$ & $-0.92 \pm 1.33$ \\
\hline $\mathrm{N}$ & 54 & 42 & 96 & 9 \\
\hline HDL-C (before treatment) (mM) & $0.96 \pm 0.22$ & $1.01 \pm 0.28$ & $0.97 \pm 0.24$ & $1.08 \pm 0.35$ \\
\hline HDL-C (after treatment) (mM) & $0.97 \pm 0.24$ & $1.01 \pm 0.38$ & $0.98 \pm 0.20$ & $1.78 \pm 0.21$ \\
\hline$\Delta \mathrm{HDL}-\mathrm{C}(\mathrm{mM})$ & $0.01 \pm 0.25$ & $0.00 \pm 0.34$ & $0.01 \pm 0.28$ & $0.10 \pm 0.22$ \\
\hline $\mathrm{N}$ & 54 & 42 & 96 & 9 \\
\hline LDL-C (before treatment) (mM) & $3.27 \pm 0.79$ & $3.06 \pm 0.76$ & $3.20 \pm 0.77$ & $3.11 \pm 0.95$ \\
\hline LDL-C (after treatment) (mM) & $2.65 \pm 0.78 * *$ & $2.23 \pm 0.69 * *$ & $2.42 \pm 0.70^{* *}$ & $2.23 \pm 0.78^{* *}$ \\
\hline$\Delta \mathrm{LDL}-\mathrm{C}(\mathrm{mM})$ & $-0.63 \pm 0.83$ & $-0.82 \pm 0.87$ & $-0.71 \pm 0.85$ & $-0.89 \pm 0.70$ \\
\hline $\mathrm{N}$ & 25 & 21 & 46 & 4 \\
\hline Lipoprotein (a) (before treatment) (mg/L) & $398.64 \pm 250.85$ & $394.72 \pm 178.23$ & $396.53 \pm 212.49$ & $465.51 \pm 324.39$ \\
\hline Lipoprotein (a) (after treatment) (mg/L) & $387.46 \pm 193.89$ & $333.14 \pm 163.73^{* *}$ & $355.26 \pm 198.73$ & $306.50 \pm 182.01$ \\
\hline$\Delta$ Lipoprotein $(\mathrm{a})(\mathrm{mg} / \mathrm{L})$ & $-11.28 \pm 184.78$ & $-61.57 \pm 86.98$ & $-34.24 \pm 149.04$ & $-159.00 \pm 165.54$ \\
\hline
\end{tabular}

\section{DISCUSSION}

It is currently believed that CHD is caused by multiple genetic factors combined with environmental factors (Tsujita et al., 2007; Kimura, 2009), and that lipid metabolism disorder can lead to the occurrence of CHD (Kolovou et al., 2011). The present study demonstrated that the plasma levels of TC, LDL-C, and lipoprotein(a) were significantly higher in the ACS and stable CHD groups compared to the control group. These blood lipid profiles have been confirmed with several independent lines of evidence (Kimura et al., 2011). The plasma level of HDL-C was lower in the ACS group than the stable CHD group, and was much lower than that of the control group. This indicates that HDL-C has a stabilizing effect on lipid plaque.

LDL-C and HDL-C contents have been shown to be closely related to the occurrence of CHD (Kuivenhoven et al., 1998; Lemieux et al., 2000; Krauss, 2001). In addition, CETP is a key enzyme in plasma lipoprotein that influences TG and HDL-C metabolism (Huang et al., 2011). Therefore, improvement of CETP activity can lead to increased TG, LDL-C, and very low-density lipoprotein cholesterol (VLDL-C) levels, and decreased HDL-C levels. The activity and transport functions of CETP are affected by genetic and environmental factors (Ghasabeh et al., 2007; López-Ríos et al., 2011). The action mechanism of CETP on atherosclerosis is complex. CETP defects or decreased activity leads to increased HDL-C levels and enlargement of LDL particles, which prevents the occurrence of atherosclerosis (Kuivenhoven et al., 1998). At the same time, decreased CETP activity may result in the accumulation of large particles of HDL and in the reduction of neogenic small and dense HDL particles. Therefore, the capacity for uptaking cholesterol from peripheral cells is decreased, which affects reverse cholesterol transport and leads to atherosclerosis (Lemieux et al., 2000; Stancáková et al., 2006). The pathological, physiological, and clinical significance of 
CETP can be more accurately evaluated by investigating the relationship between CETP and atherosclerosis from a molecular biology perspective. CETP gene expression plays a decisive role in lipid transport and lipoprotein composition. To date, more than 10 novel genotypes have been found resulting from genetic polymorphisms and mutations of the CETP gene (Wu et al., 2006), and the TaqIB polymorphism is particularly universal (Hassanzadeh et al., 2009). Based on allele differences, the TaqIB polymorphism can be divided into three genotypes: $\mathrm{B} 1 \mathrm{~B} 1, \mathrm{~B} 1 \mathrm{~B} 2$, and B2B2, corresponding to the mutant homozygote, heterozygote, and defective homozygote, respectively. Allele B1 is closely related to increases in plasma CETP levels and activity (Kuivenhoven et al., 1998), and is correlated with LDL-C levels.

In the present study, the HDL-C level was significantly higher in patients with the $\mathrm{B} 1 \mathrm{~B} 2$ genotype compared to patients with the B1B1 genotype. This is consistent with results of Kuivenhoven et al. (1998) that showed that plasma CETP activity was lower in B1B2 patients than in B1B1 patients, resulting in increased plasma HDL-C levels. Consequently, atherosclerosis could not easily occur. According to this principle, plasma HDL-C levels should be relatively higher in patients with genotype B2B2. However, no significant difference was found in any blood lipid index between B1B2 and B2B2 genotypes in the present study. One possible explanation is that the frequency of $\mathrm{B} 2 \mathrm{~B} 2$ in the population is smaller than that of the other two genotypes. Indeed, B2B2 was the least common genotype in the present sample, which might have influenced the results. Another possibility is that the expression intensity of the defective allele $\mathrm{B} 2$ is weaker than that of the mutant allele B1.

The protective effect of atorvastatin in patients with CHD has been confirmed in several studies (Boekholdt et al., 2005). In the present study, after three months of lipid lowering therapy with atorvastatin, the plasma level of HDL-C was increased in patients with all CETP TaqIB genotypes, but the difference was not significant, and there were no significant differences among the three genotypes. In addition, after treatment with atorvastatin, the plasma TG level was significantly decreased in B2B2 patients, and was lower than that of $\mathrm{B} 1 \mathrm{~B} 1$ and B1B2 patients (B1 carriers).

Due to the dependence of lipid transport and lipolysis processes on TG, the plasma TG level has become known as an important factor affecting the HDL-C level. When the concentration of TG in HDL increases to a certain degree, TG is hydrolyzed by hepatic lipase, resulting in a decrease in particle size and an increase in density. Consequently, it is rapidly cleared from plasma, leading to a reduction in the HDL-C level and an increase in the risk of CHD (Rajman et al., 1996; Hokanson et al., 1999).

The B2B2 genotype is associated with decreased CETP activity and increased HDLC levels (Yilmaz et al., 2005; Xu et al., 2006; Yijiang et al., 2008). In patients with B2B2, the plasma TG level significantly decreased after lipid-lowering therapy with atorvastatin. The CETP gene contains 16 exons and 15 introns, and TaqIB is the first intron (Corbex et al., 2000). The TaqIB polymorphism plays a key role in regulating the expression of the whole gene, which is possibly because it is a target of statins for exerting its pharmacological function. This polymorphism can cause changes in the promoter region rather than in any one amino acid (Boekholdt and Thompson, 2003), and thus affects CETP activity directly and changes the cholesterol ester transport speed, leading to a series of changes in the blood lipid profile. Although the decrease in the TG level in patients with the B2B2 genotype after treatment with atorvastatin was demonstrated in the present study, the relationship among TaqIB, statins, and blood lipid still needs further investigation. 


\section{REFERENCES}

Boekholdt SM and Thompson JF (2003). Natural genetic variation as a tool in understanding the role of CETP in lipid levels and disease. J. Lipid Res. 44: 1080-1093.

Boekholdt SM, Sacks FM, Jukema JW, Shepherd J, et al. (2005). Cholesteryl ester transfer protein TaqIB variant, highdensity lipoprotein cholesterol levels, cardiovascular risk, and efficacy of pravastatin treatment: individual patient meta-analysis of 13,677 subjects. Circulation 111: 278-287.

Corbex M, Poirier O, Fumeron F, Betoulle D, et al. (2000). Extensive association analysis between the CETP gene and coronary heart disease phenotypes reveals several putative functional polymorphisms and gene-environment interaction. Genet. Epidemiol. 19: 64-80.

Estévez-González MD, Saavedra-Santana P, López-Ríos L, Chirino R, et al. (2010). HDL cholesterol levels in children with mild hypercholesterolemia: effect of consuming skim milk enriched with olive oil and modulation by the TAQ 1B polymorphism in the CETP gene. Ann. Nutr. Metab. 56: 288-293.

Ghasabeh TH, Firoozrai M, Zonouz AE, Radmehr H, et al. (2007). One common polymorphism of cholesteryl ester transfer protein gene in Iranian subjects with and without primary hypertriglyceridemia. Pak. J. Biol. Sci. 10: 4224-4229.

Hassanzadeh T, Firoozrai M, Zonouz AE, Zavarehee A, et al. (2009). Taq1B polymorphism of cholesteryl ester transfer protein (CETP) gene in primary combined hyperlipidaemia. Indian J. Med. Res. 129: 293-298.

Hokanson JE, Brunzell JD, Jarvik GP, Wijsman EM, et al. (1999). Linkage of low-density lipoprotein size to the lipoprotein lipase gene in heterozygous lipoprotein lipase deficiency. Am. J. Hum. Genet. 64: 608-618.

Hsieh MC, Tien KJ, Chang SJ, Lo CS, et al. (2007). Cholesteryl ester transfer protein B1B1 genotype as a predictor of coronary artery disease in Taiwanese with type 2 diabetes mellitus. Metabolism 56: 745-750.

Huang Y, Wu Y, Liu R, Fan P, et al. (2011). Differential effect of ATP binding cassette transporter A1 R219K and cholesteryl ester transfer protein TaqIB genotypes on HDL-C levels in overweight/obese and non-obese Chinese subjects. Acta Cardiol. 66: 231-237.

Kimura A (2009). Does a gene polymorphism predisposing to an intermediate phenotype predict the risk of disease? A lesson from CETP, high-density lipoprotein-cholesterol and coronary heart disease. Circ. J. 73: 1016-1017.

Kimura H, Miyazaki R, Imura T, Masunaga S, et al. (2011). Smaller low-density lipoprotein size as a possible risk factor for the prevalence of coronary artery diseases in haemodialysis patients: associations of cholesteryl ester transfer protein and the hepatic lipase gene polymorphism with low-density lipoprotein size. Nephrology 16: 558-566.

Kolovou G, Vasiliadis I, Kolovou V, Karakosta A, et al. (2011). The role of common variants of the cholesteryl ester transfer protein gene in left main coronary artery disease. Lipids Health Dis. 10: 156.

Krauss RM (2001). Dietary and genetic effects on low-density lipoprotein heterogeneity. Annu. Rev. Nutr. 21: 283-295.

Kuivenhoven JA, Jukema JW, Zwinderman AH, de Knijff P, et al. (1998). The role of a common variant of the cholesteryl ester transfer protein gene in the progression of coronary atherosclerosis. The regression growth evaluation statin study group. N. Engl. J. Med. 338: 86-93.

Lemieux I, Pascot A, Dumont M and Brochu M (2000). Is the high triglyceride-low HDL cholesterol atherogenic dyslipidemia a small, dense LDL or HDL phenotype? Atherosclerosis 151: 98-102.

López-Ríos L, Pérez-Jiménez P, Martínez-Quintana E, Rodriguez GG, et al. (2011). Association of Taq1B CETP polymorphism with insulin and HOMA levels in the population of the Canary Islands. Nutr. Metab. Cardiovasc. Dis. 21: 18-24.

Pillois X, Phuong Do TN, Reynaud A, Benchimol D, et al. (2009). TaqIB polymorphism in cholesterol ester transfer protein (CETP) gene predicts future cardiovascular death in patients experiencing an acute coronary syndrome. Clin. Chem. Lab. Med. 47: 1039-1046.

Rajman I, Kendall MJ, Cramb R, Holder RL, et al. (1996). Investigation of low density lipoprotein subfractions as a coronary risk factor in normotriglyceridaemic men. Atherosclerosis 125: 231-242.

Ranjith N, Pegoraro RJ and Rom L (2009). Lipid profiles and associated gene polymorphisms in young Asian Indian patients with acute myocardial infarction and the metabolic syndrome. Metab. Syndr. Relat. Disord. 7: 571-578.

Stancáková A, Baldaufová L, Javorský M, Kozárová M, et al. (2006). Effect of gene polymorphisms on lipoprotein levels in patients with dyslipidemia of metabolic syndrome. Physiol. Res. 55: 483-490.

Tsujita Y, Nakamura Y, Zhang Q, Tamaki S, et al. (2007). The association between high-density lipoprotein cholesterol level and cholesteryl ester transfer protein TaqIB gene polymorphism is influenced by alcohol drinking in a population-based sample. Atherosclerosis 191: 199-205.

Wu Y, Bai H, Liu R, Liu Y, et al. (2006). Analysis of cholesterol ester transfer protein gene TaqIB and -629 C/A polymorphisms in patients with endogenous hypertriglyceridemia in Chinese population. Zhonghua Yi Xue Yi Chuan Xue Za Zhi. 23: 640-646. 
Yijiang Z, Ruixing Y, Yiyang L, Yaju D, et al. (2008). Association of TaqIB polymorphism in the cholesteryl ester transfer protein gene with serum lipid levels in the Guangxi Hei Yi Zhuang and Han populations. J. Investig. Med. 56: 847-857.

Yilmaz H, Isbir T, Agachan B and Karaali ZE (2005). Effects of cholesterol ester transfer protein Taq1B gene polymorphism on serum lipoprotein levels in Turkish coronary artery disease patients. Cell Biochem. Funct. 23: 23-28.

Xu ZH, Guo HW and Huang ZY (2006). Effects of cholesterol ester transfer protein Taq1B polymorphism on response of serum HDL-C to dietary factors in hyperlipidemia patients. Zhonghua Yu Fang Yi Xue Za Zhi. 40: 269-272. 